\title{
Network ARCHITECTURE AND PERFormanCE ANALYSIS OF MULTI-OLT PON FOR FTTH AND WIRELESS SENSOR NETWORKS
}

\author{
Monir Hossen and Masanori Hanawa \\ Interdisciplinary Graduate School of Medicine and Engineering \\ University of Yamanashi, Japan \\ mnr.hossen@gmail.com, hanawa@ieee.org
}

\begin{abstract}
An integrated fiber-to-the-homes (FTTHs) and wireless sensor network (WSN) provides a cost-effective solution to build up an immaculate ubiquitous-City (U-city). The key objectives of effective convergence of FTTH and WSN are less computational complexity for data packet processing, low installation cost, and good quality of services. In this paper, we introduce an integrated network structure of multi-optical line terminal (multi-OLT) passive optical network (PON) which can accommodate multiple service providers in a single PON. A modified version of interleaved polling algorithm is proposed for scheduling of control messages from multiple OLTs in a single network. We also provide detailed numerical analysis of cycle time variation, successive grant scheduling time, and average packet delay for both uniform and non-uniform traffic loads generated by each ONU, using fixed service bandwidth allocation scheme and limited service bandwidth allocation scheme. We also compare the throughput of the proposed scheme with existing single-OLT PON for non-uniform traffic load using limited service bandwidth allocation scheme. The simulation results show that the proposed multi-OLT PON system can supports existing bandwidth allocation schemes with better performance than the single-OLT PON in terms of average packet delay, bandwidth utilization, and throughput.
\end{abstract}

\section{KEYWORDS}

Multi-OLT PON, Network Architecture, WSN, FTTH, Limited Service Bandwidth Allocation Scheme, Interleaved Polling Algorithm, Non-uniform Traffic, Packet Delay

\section{INTRODUCTION}

Currently, the network structure of a u-City is very complicated due to convergence of several new service providers. WSN is also one of the most important networks to build up a perfect uCity. Day-by-day the diameter of WSN becomes very large to provide the numerous new facilities and supports. Due to the large diameter as well as large number of wireless hops from the personal area network coordinator (PANC) to the surface nodes of a WSN, data packets from the surface nodes suffer from excess time delay. To solve this problem, clustering of large WSN is a good approach to reduce the individual network diameter as well as number of hops while the whole network size is enlarged. In the clustered WSN, every cluster consists of a cluster head $(\mathrm{CH})$, and data from all nodes in a cluster are transmitted to a $\mathrm{CH}$ over a short distance with a small number of hops. So the data transmission delay in the clustered sensor network will be much lower than the case when each node directly communicates with the PANC through a large number of wireless hops. Even though, the main focuses of this paper is only on latency issue and analysis of energy efficiency is out of scope of this paper but the energy efficiency is another significant factor for an efficient WSN. Because the capacity of battery inside every sensor node and recurrent replacement of them is unrealistic that is why preservation of that energy is very important [1]. Some clustering algorithms of WSN have been developed to provide the energy efficiency. In [2], an energy efficient homogeneous clustering 
algorithm is proposed to extend the lifetime of sensor nodes and to maintain a balance energy consumption of nodes of a sensor networks.

Since in a u-city all the information systems are virtually linked together, it is very important to make a linkup with optical network terminals and WSNs. In [3, 4], a cluster based WSN is proposed where all sensor nodes of a cluster is connected with a $\mathrm{CH}$ through a small number of wireless hops and all the CHs are connected to a PANC by Radio-over- Fiber (RoF) links. Application of optical network in clustered WSN is very useful in that the optical attenuation is very small while wide bandwidth can be utilized.

PON is an optical network it does not contain active elements from source to destination which effectively reduces the active power consumption of the networks. PON provides several advantages over other access technologies. The main advantages of PON are high data rate, easy adaptability with new protocols and services, simple network structure, minimize the fiber deployment, less maintenance, and allow for long distance between the central office and customer premises. PON also provides effective solutions to satisfy the increasing capacity demand in the access part of the communication arena. In [5], a clustered WSN is proposed where all the $\mathrm{CHs}$ are connected with the PANC through a PON system. However, convergence of data networks and sensor networks in a single-OLT PON will increase the computational complexity for data packet processing in the OLT. To mitigate this problem some polling algorithms have been proposed to allow additional time in OLT for computation and management in addition with the guard time between every two successive ONUs [6]. Due to these increased computational complexity and additional computation time, some delay sensitive traffic of WSN will suffer from unexpected delay. To solve this problem, a single PON structure with multiple OLTs can be a good candidate.

In this paper, we propose a converged network structure of multi-OLT PON for FTTH terminals and clustered-based WSN. Here, we assume that a tree structured PON consists of two OLTs in the root side and $N$ ONUs in the leave side sharing the same optical fiber links. One OLT is connected with all the FTTH terminals, and the other is connected with all the CHs of WSN. Although this system requires one additional OLT, it is inexpensive comparing with expenses for deploying two separate networks of data and WSN. We also propose a modified version of the interleaved polling algorithm [6], and scheduling algorithm for control messages for the multi-OLT PON system. The simulation results for successive grant scheduling time, evolution of cycle time, and average packet delay for both uniform and non-uniform traffic conditions are investigated and compared with a single-OLT PON for fixed service (FS) bandwidth allocation scheme and limited service (LS) bandwidth allocation scheme. Throughput of multi-OLT PON is also analysed and compared with the single-OLT PON for non-uniform traffic load considering LS bandwidth allocation scheme. Since generated packet sizes of FTTH terminals and CHs of WSN are not same, a comparative analysis is shown by changing the ratio of maximum bandwidth for FTTH and WSN because the maximum cycle time, $T_{\max }$, is constant for PON system.

The rest of this paper is organised as follows. Section 2 presents the related works. In section 3, we presents the network architecture of the proposed multi-OLT PON. In section 4, we present a modified version of the interleaved polling algorithm, and a scheduling algorithm of a control message for multi-OLT PON. Section 5 shows the simulation results. Finally, section 6 draws conclusions.

\section{RELATED WORKS}

In this section, we describe some existing hybrid network architecture of WSN and FTTH terminals, interleaved polling algorithm, and bandwidth allocation algorithms for single-OLT PON system. 
In the clustered WSN, usually all $\mathrm{CHs}$ are responsible to transmit data packet to the PANC through radio frequency (RF) transmission which suffers from data collision and more active energy consumption. However, conservation of energy in WSN is one of the prime requirements. To achieve this, J. Tang et al [3] proposed hybrid RoF based WSN architecture to combat the existing problems and to enhance the radio coverage and quality of service (QoS) for WSN. Here, whole sensor network is divided into several clusters and each cluster contains a static $\mathrm{CH}$. All static $\mathrm{CHs}$ of WSN are distributed randomly in a wide area to collect the environment data such as temperature, humidity, the location of workers in the mine etc. Finally, all of the CHs are connected with the remote antenna units (RAUs) of RoF system to avoid data collision and RF transmission from $\mathrm{CHs}$ to PANC.

In $[5,7]$ a PON based WSN is proposed where synchronous latency secured (Sync-LS) medium access control (MAC) protocol and cooperative clustering algorithm are used to reduce the latency of a large sensor network. MAC protocols play a vital role for efficient data transmission and collision avoidance in wireless communication systems. Both of the papers provide latency efficiency in the wireless part of PON based WSN due to the cooperative clustering algorithm and Sync-LS MAC protocol using existing PON algorithms. But all these proposed algorithms are designed for single-OLT PON system where additional computational complexity of data packet processing in the OLT due to the convergence of data network terminals and WSN are not considered.

Interleaved polling algorithm [6] is a widely used algorithm in which OLT polls the ONUs individually and issues Grants to them in a round-robin fashion. In this algorithm OLT maintains a polling table containing the number of bytes waiting in each ONU's buffer and the round-trip-time (RTT) to each ONU. At the end of a transmission window, every ONU informs its queue size to the OLT by Report message. Interleaved polling algorithm has been developed to implement a dynamic bandwidth allocation scheme to improve the bandwidth utilization in PON system. The main principle of this algorithm is allocation of bandwidth to ONUs to avoid data collision and to fairly share the channel capacity. While this scheme is effective to avoid data collision, but in the single-OLT PON, it must requires some guard time between every two successive ONUs to avoid data overlapping.

In PON systems, each ONU's upstream bandwidth is decided by allocated time slots specified by the OLT in unit time [8]. Bandwidth allocation algorithm has a major impact on minimizing latency, improving fairness, meeting quality of services guaranties, and requirement of buffer size in upstream direction. In general sense, bandwidth allocation algorithms can be classified into two major groups; fixed bandwidth allocation and dynamic bandwidth allocation (DBA) algorithms.

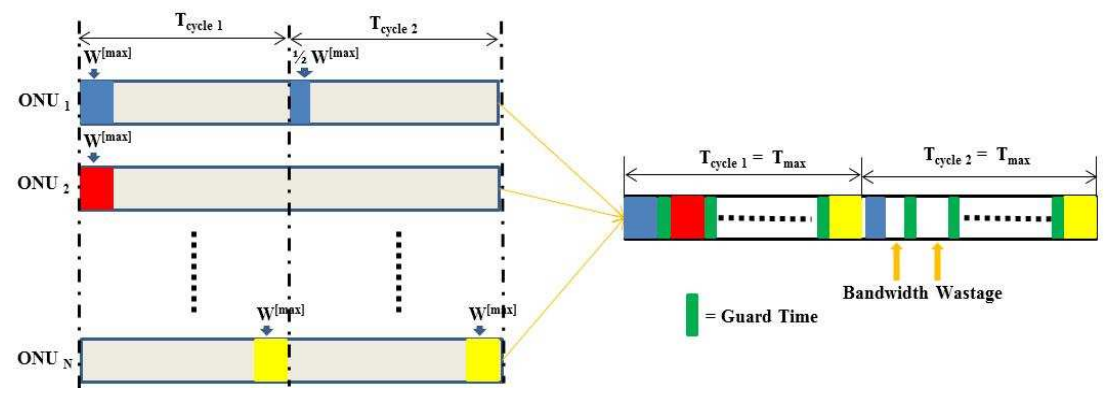

Figure 1. Fixed bandwidth allocation scheme

In [9], the performance of EPON using a fixed bandwidth allocation algorithm is studied where all traffics are considered to a single class. This scheme is simple and always grants the maximum window to all ONUs, as a result the cycle time $T_{\text {cycle }}$ is constant for all traffic loads as 
shown in Figure 1. The main drawback of this algorithm is light-loaded ONUs will under-utilize their allocated bandwidth leading to increased delay to other ONUs and eventually deteriorate the throughput and bandwidth utilization of the system.

DBA schemes provide flexible bandwidth sharing of allocation among users. DBA is suitable for burst traffic such as FTTHs and VoIP. Different DBA algorithms have been developed to improve the bandwidth utilization and to adopt with the current demand of huge traffic. In the LS scheme $[6,10]$, the time-slot length of each ONU is upper bounded by the maximum bandwidth, $W^{[\max ]}$. When the requested bandwidth by the ONU is less than $W^{[\max ]}$, the OLT grants the requested bandwidth; otherwise, $W^{[\max ]}$ is granted. Figure 2 shows the LS bandwidth allocation scheme. Since the granted window is based on the requested window, the cycle time $T_{\text {cycle }}$ is variable. As shown in the figure, cycle time for first cycle is $T_{\text {cycle } 1}=T_{\max }$ because every ONU requests for maximum bandwidth $W^{[\max ]}$. On the other hand, cycle time in the $2^{\text {nd }}$ cycle is $T_{\text {cycle } 2}=T_{\max }-T_{S}$, here, $T_{S}$ is the cycle time saving due to light-loaded ONUs. This scheme reduces the bandwidth wastage by granting smaller bandwidth to the light-loaded ONUs. However, one limitation of this algorithm is that making $T_{\text {cycle }}$ too small will result lower bandwidth utilization because of constant guard time for every two successive ONUs.

In [11], a new DBA is proposed where ONUs in the network are divided into two sets, one set contains the ONUs with bandwidth guaranteed services while the second set contains the ONUs with best effort services. This scheme is able to provide guaranteed bandwidth for premier subscribers while best effort services are provided to other subscribers. However, all of these DBA algorithms are proposed only for the single-OLT PON and must require guard time between every two successive ONUs to avoid data overlapping. Due to this guard time, some bandwidth wastage problem is observed.

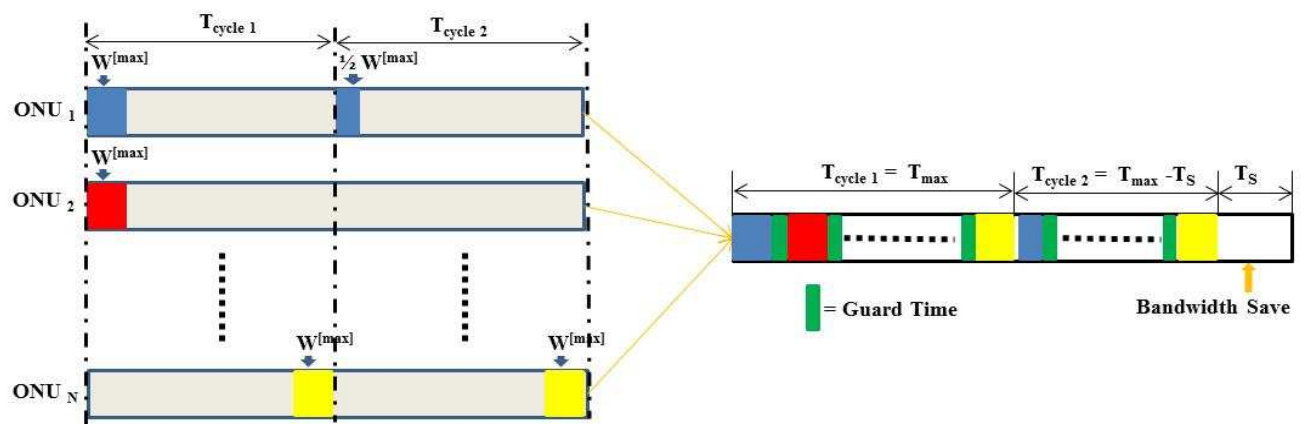

Figure 2. Dynamic bandwidth allocation scheme

\section{Network ArChitecture Of MULTI-OLT PON}

In this paper, we consider an access optical network architecture consisting of two OLTs and $N$ ONUs connected using a single PON structure as shown in Figure 3. For simplicity, only two OLTs, OLT1 and OLT2/PANC, and four ONUs with tree based network topology are shown in the figure to explain the network structure and data transmission sequences for both upstream and downstream.

All transmissions in the proposed multi-OLT PON are performed between two OLTs in the root side and four ONUs in the leaf side of the tree topology. Here, OLT1 is connected with FTTH data terminals, ONU1 and ONU3. On the other hand, OLT2/PANC is connected with the static CHs of WSN, ONU2 and ONU4. All the connections between OLTs and ONUs are established through optical fibers and a passive splitter/combiner. 
In downstream transmission, both OLTs follow the same polling table to initiate a transmission of a Grant message to the ONUs. Depending on the RTT delay between OLTs and ONUs, the $1^{\text {st }}$ Grant message can be scheduled by any of both OLTs, because RTT depends on the physical distances between OLTs and ONUs and these distances are not fixed for all ONUs. Since, all downstream transmissions are broadcasted (point to multi-point transmission) from OLT to ONUs, both OLTs broadcast their Grant messages through an optical splitter and each ONU filters the received packets according to its destination address.

In upstream transmission, all ONUs share a common channel capacity and resources, and various multiple access schemes are exist to share a common channel in a PON-based access network. In our model, we consider time-division multiple access (TDMA) scheme to ensure the use of a single upstream wavelength for all users and a single receiver in the head end to reduce the system cost. According to the principle of the proposed multi-OLT PON system, OLT1 accepts data only from the ONUs of FTTH terminals (ONU1, ONU3) and OLT2 accepts data only from the ONUs of WSN (ONU2, ONU4).

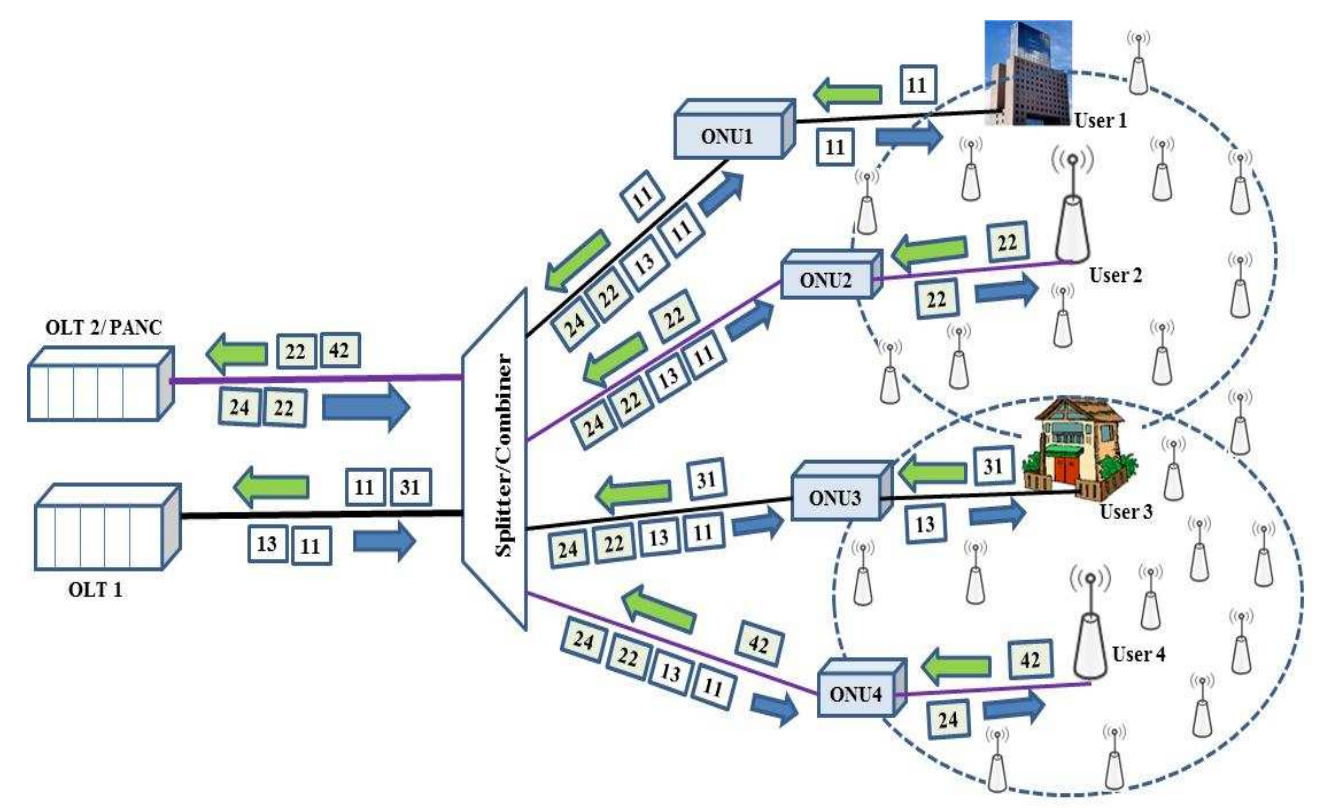

Figure 3. Network architecture and data transmission in proposed multi-OLT PON

\section{Modified Algorithms For Multi-OLT PON}

In this section, the modified version of interleaved polling algorithm and scheduling algorithm of a control message suitable for the multi-OLT PON system are described.

\subsection{Interleaved polling algorithm}

The main aspect of polling algorithm is the scheduling of data transmission of ONUs. Usually, a polling protocol is cycle based, which used to avoid high traffic load as well as data collision, and it limits the maximum transmission window for each ONU. A commonly used polling algorithm is round-robin, which orders the transmission of every ONU periodically. To improve the network performance, interleaved polling with adaptive cycle time (IPACT) [6] is used. Interleaved polling algorithm can also have different policies, such as interleaved polling algorithm with and without stop polling. In all interleaved polling algorithms, an OLT contains a polling table which provides information about the RTT to every ONU and their granted 
window size. The knowledge of RTT and granted window size from the polling table are used to avoid data collision and overlapping of packets from different ONUs. Additionally, a guard time is used between every two successive ONUs to avoid overlapping of transmission windows by fluctuations in RTT of different ONUs [12] and on/off timing of laser of OLT and ONUs. Without using this guard time a single-OLT PON cannot ensure the overlap free transmission between two successive ONUs.

The data transmission from multiple OLTs and ONUs also must be well scheduled to improve network performance and to avoid data collision. A modified version of the interleaved polling algorithm [6] is used for the proposed multi-OLT PON system, where a common polling table is considered for both of the OLTs. Figure 4 shows the modified interleaved polling algorithm and RTT calculation procedure for the proposed multi-OLT PON system. For simplicity, ONU 1, 3, $5 \ldots(2 i-1)(i$ is integer) are for the FTTH terminals and those are connected with OLT1. On the other hand, ONU 2, 4, $6 \ldots 2 i$ are for the CHs of WSN and those are connected with OLT2.

In the multi-OLT PON, no guard time is required, because data of every two successive ONUs will be received by two different OLTs. So there is no possibility of data overlapping due to fluctuations of laser on/off timing and RTT. After receiving of data from a particular ONU, every OLT gets enough time before receiving data from the next ONU. This way, packet delay of the network and computational complexity of OLTs can be decreased while bandwidth utilization will be increased.

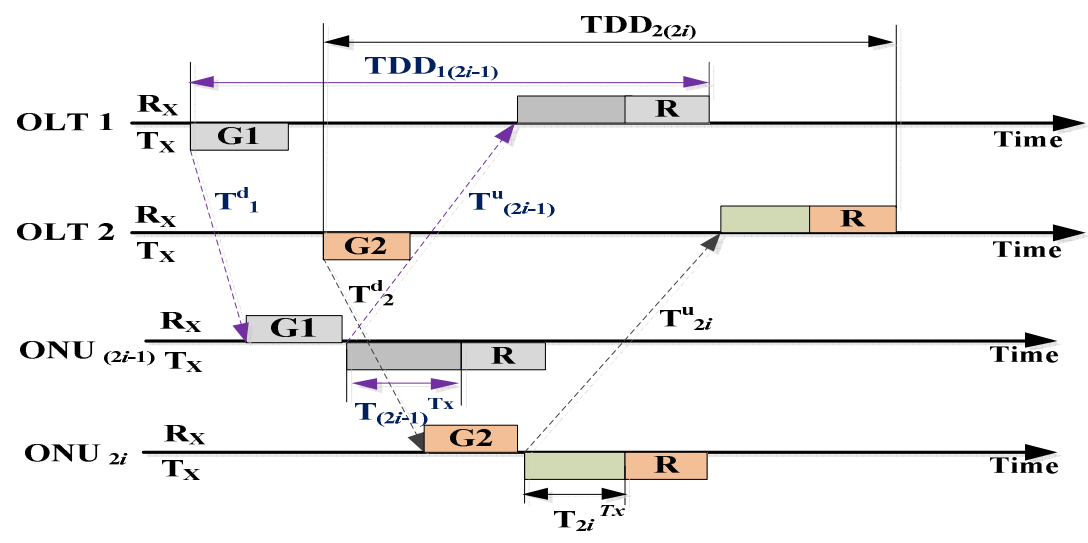

Figure 4. RTT and data transmission time in multi-OLT PON

Equations (1) and (2) represent the principle of RTT calculation for OLT1 and OLT2, equations (3) and (4) represent data transmission delay for OLT1 and OLT2, respectively.

$$
\begin{aligned}
& R T T_{1(2 i-1)}=T_{1}^{d}+T_{(2 i-1)}^{u} \\
& R T T_{2(2 i)}=T_{2}^{d}+T_{2 i}^{U} \\
& T D D_{1(2 i-1)}=T_{G}+T_{1}^{d}+T_{(2 i-1)}^{T x}+T_{(2 i-1)}^{u}+T_{R} \\
& T D D_{2(2 i)}=T_{G}+T_{2}^{d}+T_{2 i}^{T x}+T_{2 i}^{u}+T_{R}
\end{aligned}
$$

where, $T_{G}$ and $T_{R}$ are the transmission times of Grant and Report messages, $T^{d}$ is the downstream propagation delay, $T^{T x}$ is the upstream transmission time of data, and $T^{u}$ is the upstream propagation delay.

Transmission time and propagation delay of data depend on the data transmission speed of the PON and physical distance between OLTs and ONUs. Usually, physical distance between an 
International Journal of Wireless \& Mobile Networks (IJWMN) Vol. 3, No. 6, December 2011

OLT and ONUs are not equal [13] but the data transmission speed is a constant for TDMA PON. In TDMA PON, downstream traffic is broadcasting in nature which is handled by OLTs and upstream traffic from ONUs is allowed at a particular transmission time [14]. In our analysis, random distances between OLTs and ONUs but constant transmission speed are considered.

\subsection{Scheduling algorithm of a control message}

During upstream transmission in a single-OLT PON, all ONUs share a single uplink optical fiber trunk connected with an OLT. To prevent data collision due to multiple ONUs transmitting at the same time, a multi-point control protocol (MPCP) is being developed [13]. Usually, the MPCP operation in PON requires two control messages, Grant and Report. A Report message contains current queue length of each ONU to inform the OLT. On the other hand, the OLT assigns a timeslot to an ONU by a Grant message. Scheduling of Grant messages from OLT depends on RTT and granted transmission window size of ONUs [6]. This is because of the variation of RTT and granted window size for different ONUs.

Figure 5 shows a scheduling diagram of control messages for the proposed multi-OLT PON system. As the scheduling of the Grant messages depend on the RTT and granted window size of different ONUs, the starting Grant message can be sent by any of the both OLTs.

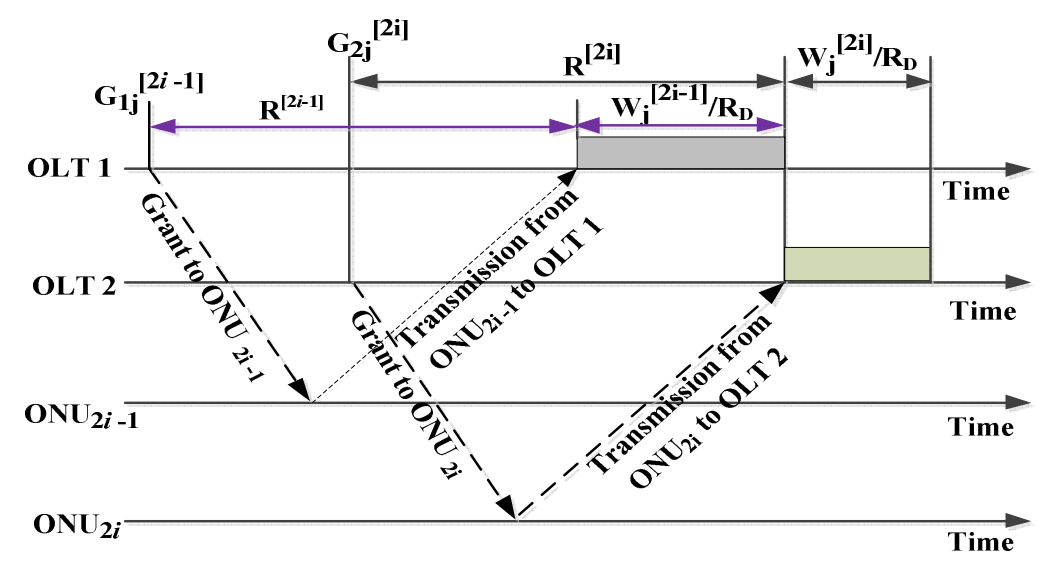

Figure 5. Scheduling diagram of control messages in multi-OLT PON

The scheduling of Grant messages as shown in above figure can be expressed as the following equations:

$$
\begin{gathered}
G_{2 j}^{[2 i]}=G_{1 j}^{[2 i-1]}+R^{[2 i-1]}-R^{[2 i]}+\frac{W_{j}^{[2 i-1]}}{R_{D}} \\
G_{1 j}^{[2 i+1]}=G_{2 j}^{[2 i]}+R^{[2 i]}-R^{[2 i+1]}+\frac{W_{j}^{[2 i]}}{R_{D}}
\end{gathered}
$$

where, $G_{2 j}^{[2 i]}$ and $G_{1 j}^{[2 i+1]}$ are the time epochs for OLT2 and OLT1 when $j^{\text {th }}$ Grant to $\mathrm{ONU}_{2 i}$ and $\mathrm{ONU}_{2 i+1}$ are transmitted respectively, $G_{1 j}^{[2 i-1]}$ is the time epoch for OLT1 when $j^{\text {th }}$ Grant to $\mathrm{ONU}_{2 i-1}$ is transmitted, $R^{[2 i-1]}$ and $\mathrm{R}^{[2 i]}$ are the RTT from OLT1 to $\mathrm{ONU}_{2 i-1}$ and OLT2 to $\mathrm{ONU}_{2 i}$ respectively, $R^{[2 i+1]}$ is the RTT from OLT1 to $\mathrm{ONU}_{2 i+1}, W_{j}^{[2 i-1]}$ and $W_{j}^{[2 i]}$ are the $j^{\text {th }}$ window size for $\mathrm{ONU}_{2 i-1}$ and $\mathrm{ONU}_{2 i}$ respectively, and $R_{D}$ is the transmission speed. 


\section{Performance Evaluations}

In this section, the system performances of the proposed multi-OLT PON with the FS bandwidth allocation scheme and the LS bandwidth allocation scheme are analysed and compared with the existing single-OLT PON in terms of successive Grant scheduling time, cycle time evolution, and average packet delay for uniform and non-uniform traffic loads. Finally, we have investigated the throughput of the multi-OLT PON and compared with the single-OLT PON for non-uniform traffic load and LS bandwidth allocation scheme. For the variation of traffic model (considering maximum packet size) between FTTH terminals and CHs of WSN, performances of the multi-OLT PON system are also analysed using different packet sizes for FTTH terminals and WSN by considering different maximum granted window sizes, $W^{[\max ]}$.

The random packet-based simulation model was used considering tree topology based PON architecture with two OLTs and 16 ONUs, where eight ONUs (ONU1, ONU3 ... ONU15) were considered for OLT1 and other eight ONUs (ONU2, ONU4 ... ONU16) were for OLT2. The distances from OLTs to ONUs were assumed as random to range from 10 to $20 \mathrm{~km} \mathrm{[15].} \mathrm{The}$ downstream transmission and upstream transmission speeds of both were $1 \mathrm{~Gb} / \mathrm{s}$. Highly bursty random traffic patterns were generated for non-uniform traffic condition. For uniform traffic condition every ONU was considered to generate an offered load while for non-uniform traffic condition every ONU was considered to generate any value from 0 to the offered load. Our simulations took into account queuing delay, transmission delay, propagation delay, and processing delay. The simulation scenario is summarized in Table 1.

Table 1. Simulation scenario.

\begin{tabular}{|l|l|l|}
\hline Symbol & Explanation & Value \\
\hline$N_{\text {ONU }}$ & Total Number of ONUs & 16 \\
\hline$N_{F T T H}$ & Number of ONUs for FTTH terminals & 8 \\
\hline$N_{W S N}$ & Number of ONUs for WSN & 8 \\
\hline$N_{O L T}$ & Number of OLTs & 2 \\
\hline$D$ & Distance between OLTs and ONUs (random) & $10-20 \mathrm{~km}$ \\
\hline$T_{\max }$ & Maximum cycle time & $2 \mathrm{~ms}$ \\
\hline$T_{g}$ & Guard time for single OLT PON & $5 \mu \mathrm{s}$ \\
\hline$R_{D}$ & Transmission speed & $1 \mathrm{~Gb} / \mathrm{s}$ \\
\hline$T_{p r o c}$ & Processing time & $10 \mu \mathrm{s}$ \\
\hline$B$ & Packet size & 1500 byte $(\mathrm{FTTH})$ \\
& & 1024 byte $(\mathrm{WSN})$ \\
\hline$B_{\text {eth }}$ & Ethernet overhead & 304 bits \\
\hline$B_{\text {rep }}$ & Report message size & 576 bits \\
\hline$P_{\max }$ & Maximum transmission window & 20 packets \\
\hline$W^{[\max ]}$ & Maximum granted window & $\left(B_{\text {eth }}+B\right)^{*} P_{\max }$ \\
\hline
\end{tabular}

Firstly, the impact of modified version of interleaved polling algorithm on the proposed multiOLT PON is investigated. In the simulation, random packet sizes but not larger than $W^{[\max ]}$ are 
generated to every ONU for the LS scheme, however, it is fixed to $W^{[\max ]}$ for every ONU with the FS scheme even if the network load is low. For simplicity, we assume that the starting time of the $1^{s t}$ Grant message by OLT1 to ONU1 is at $0.0 \mu \mathrm{s}$. Figure 6 shows the comparison of successive Grant scheduling time between the single-OLT PON and the multi-OLT PON for both of the FS and the LS bandwidth allocation algorithms. From these results, it is clear that successive Grant scheduling time of the multi-OLT PON with interleaved polling algorithm provides about $80 \mu$ s less time delay at the $16^{\text {th }}$ ONU for both of the LS scheme and the FS scheme, because the proposed multi-OLT PON does not require any guard time between every two successive ONUs.

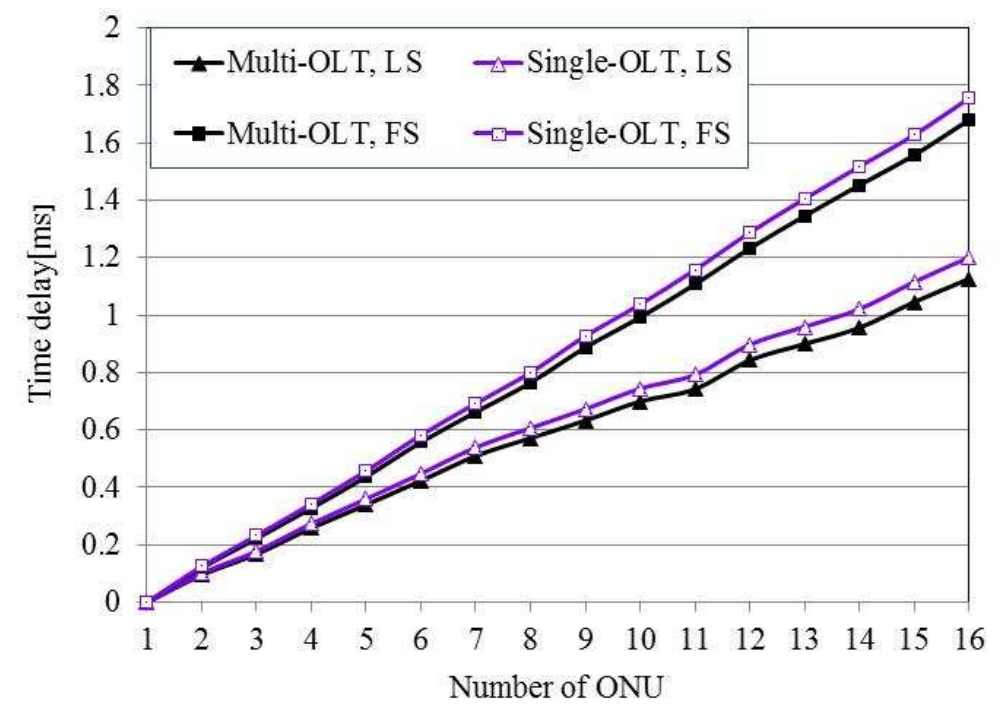

Figure 6. Comparison of successive Grant scheduling time between the single OLT PON and the multiOLT PON systems

Cycle time is an important parameter to calculate packet delay in PON system. Usually, data packets of all ONUs of present cycle are sent to the OLT in the next cycle, because all ONUs still have to inform the OLT for their queue status during the present cycle. Depending on the traffic generation time in an ONU, transmission starting delay will be on average half of a cycle time, $T_{\text {cycle }}$.

For the FS bandwidth allocation scheme, cycle time $T_{\text {cycle }}$ will be constant for all traffic loads [16]. In this case, data will suffer from the same delay in every cycle and it does not depend on the present network traffic. From the Table 1, it is clear that the cycle time for the FS scheme is always $2.0 \mathrm{~ms}$. If the traffic load is very low then bandwidth utilization problem [17] will be severe for the FS scheme due to the bandwidth wastage, Ethernet overhead, and guard time in the single-OLT PON. However, the proposed multi-OLT PON can provide better bandwidth utilization under the same traffic condition due to the avoidance of guard time. The following formula represents the cycle time for the multi-OLT PON with the FS scheme.

$$
T_{\text {cycle }}=\frac{N \cdot B_{\text {rep }}+N \cdot\left(B+B_{e t h}\right) \cdot P_{\max }}{R_{D}}
$$

where, $B_{\text {rep }}, B$, and $B_{\text {eth }}$ denote the Report message size, packet size, and Ethernet overhead respectively, $N$ represents the number of ONUs, $P_{\max }$ indicates the maximum transmission window, and $R_{D}$ is the transmission speed. 
In the LS bandwidth allocation scheme, cycle time is variable under low and non-uniform traffic condition, since the granted window size is based on the requested window size. Bandwidth utilization in the LS scheme will be improved than the FS scheme for both of the single-OLT and multi-OLT PONs due to avoidance of bandwidth wastage. Equation (8) represents the cycle time for the multi-OLT PON with the LS scheme.

$$
T_{\text {cycle }}=\frac{N \cdot B_{\text {rep }}+\sum_{i=1}^{N}\left(B+B_{\text {eth }}\right) \cdot P_{i}}{R_{D}}
$$

where, $P_{i}$ is the granted transmission window for $\mathrm{ONU}_{i}$ and $P_{i} \leq P_{\max }$.

To calculate the average packet delay in the LS scheme, consideration of the bursty nature of cycle time is important, because it is clear that the maximum queuing time of aggregated packets in an ONU depends on the cycle time of the previous cycle. Figure 7 gives an idea about the bursty nature of the cycle time for the LS scheme under non-uniform traffic condition for both of the single-OLT and the multi-OLT PONs. However, the cycle time for FS bandwidth allocation scheme is constant. The figure also clearly shows the existence of longer and shorter cycle times. Since the cycle times of previous cycles influence the data packet delay of present cycle, consideration of this cycle time variation influence the end-to-end packet delay and data queuing time in ONUs.

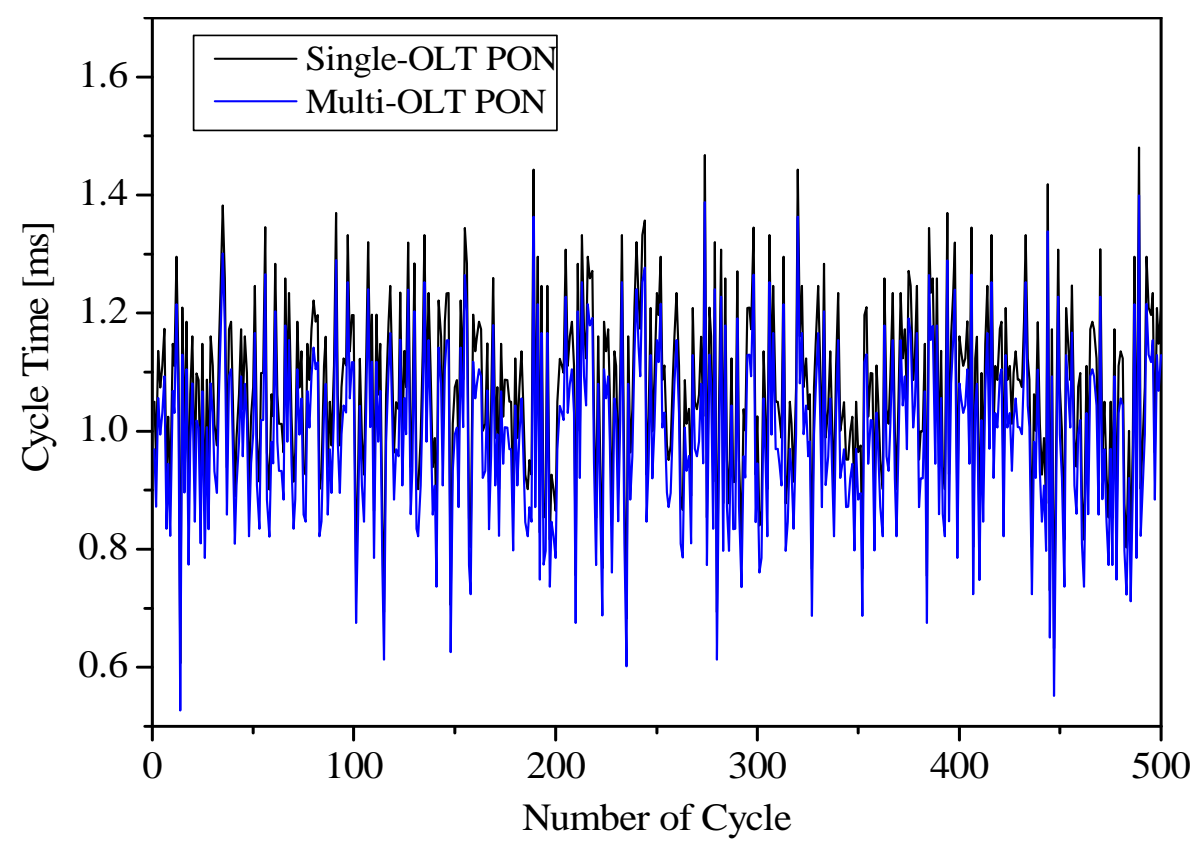

Figure 7. Evolution of cycle times for the LS scheme

Figure 8 compares the average end-to-end packet delay vs. offered load among the single-OLT PON and the proposed multi-OLT PON under uniform traffic conditions. In this simulation, same packet length is considered for both of FTTH terminals and CHs of WSN. The results show that delay characteristics are similar among the single-OLT PON and the multi-OLT PON for both of the FS scheme and the LS scheme. Small amounts of delay improvement about $<0.1 \mathrm{~ms}$ at low traffic condition and $<0.2 \mathrm{~ms}$ at high traffic condition are observed for the multi- 
OLT PON due to the avoidance of guard time. Therefore, the multi-OLT PON can support the existing FS scheme and LS scheme with better delay efficiency than the single-OLT PON.

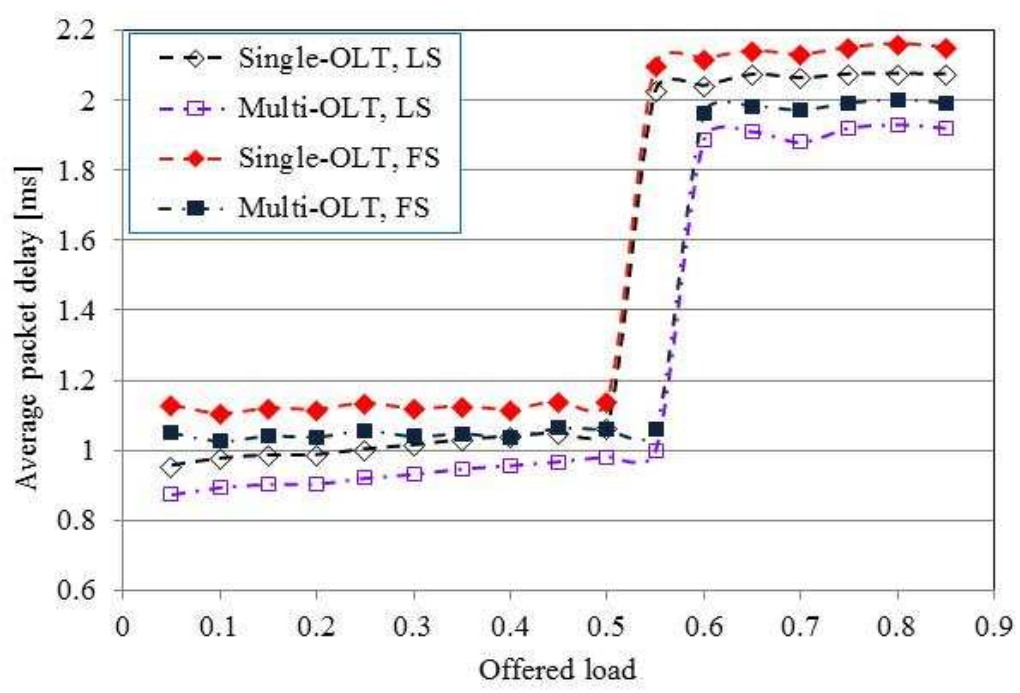

Figure 8. Comparison of average packet delay for uniform traffic load

Figure 9 compares the average end-to-end packet delay vs. offered load between the single-OLT PON and the multi-OLT PON for the LS scheme under non-uniform traffic conditions. For the single-OLT PON, the average packet delay increases sharply when the offered load changes from 0.5 to 0.55 , whereas for the multi-OLT PON, such a sharp increase of the average packet delay is observed at the offered load from 0.6 to 0.65 . The reason is similar with the case of uniform traffic load in Figure 8, and the proposed multi-OLT PON provides better delay efficiency than the single-OLT PON even under non-uniform traffic conditions.

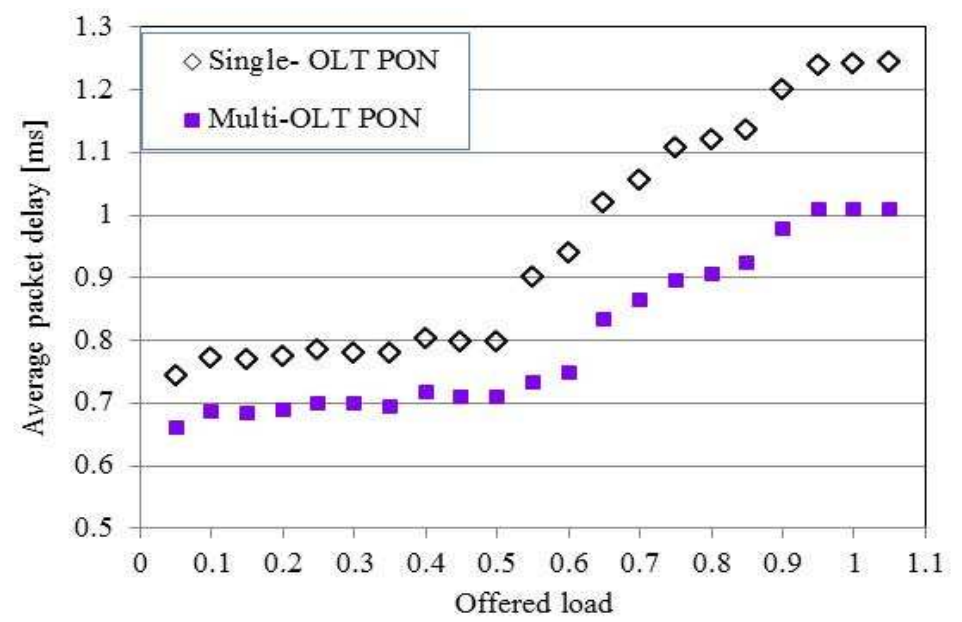

Figure 9. Comparison of average packet delay for non-uniform traffic load with the LS scheme

From the analysis of end-to-end packet delays for both of uniform and no-uniform traffic conditions it is clear that for higher traffic loads the average packet delay increases very sharply for both of the single-OLT and multi-OLT PONs. Because the aggregated traffic load becomes the determining factor and end-to-end packet delay increases quickly due to congestion. Due to the congestion, if a packet cannot be sent in its first requested transmission window, it will have an influence over two or multiple cycle times. 
By now, considering same packet length for FTTH terminals and CHs of WSN, the multi-OLT PON presented here achieves better delay efficiency than the single-OLT PON. The maximum packet length for FTTH terminals and WSN are 1500 bytes [16] and 1024 bytes [18] respectively. When same bandwidth (50\% bandwidth ratio) is allocated for both networks, then the networks will suffer from different average packet delays at the offered load $\geq 0.55$ to $\leq 0.75$ (difference of offered load is 0.2) as shown in Figure 10. Here, Figure 10 shows the average end-to-end packet delay vs. offered load characteristics under uniform traffic conditions using different bandwidth allocation ratios among FTTH terminals and CHs of WSN. To reduce this difference, we should choice the proper bandwidth allocation ratio among FTTH terminals and CHs of WSN. Similarly, if 70\% and 30\% bandwidth are used for FTTH terminals and CHs of WSN respectively then the difference of offered load becomes $0.3(\geq 0.45$ to $\leq 0.75)$. On the other hand, if $60 \%$ and $40 \%$ bandwidth ratio is used then the difference of offered load reduced to 0.05 ( $\geq 0.6$ to $\leq 0.65$ ). Considering these cases, $60 \%$ and $40 \%$ bandwidth for FTTH terminals and CHs of WSN respectively provide optimized average packet delay for both networks. This bandwidth ratio reflects the ratio of packet length of FTTH terminals and WSN if the packet length is changed then the bandwidth ratio will also be changed.

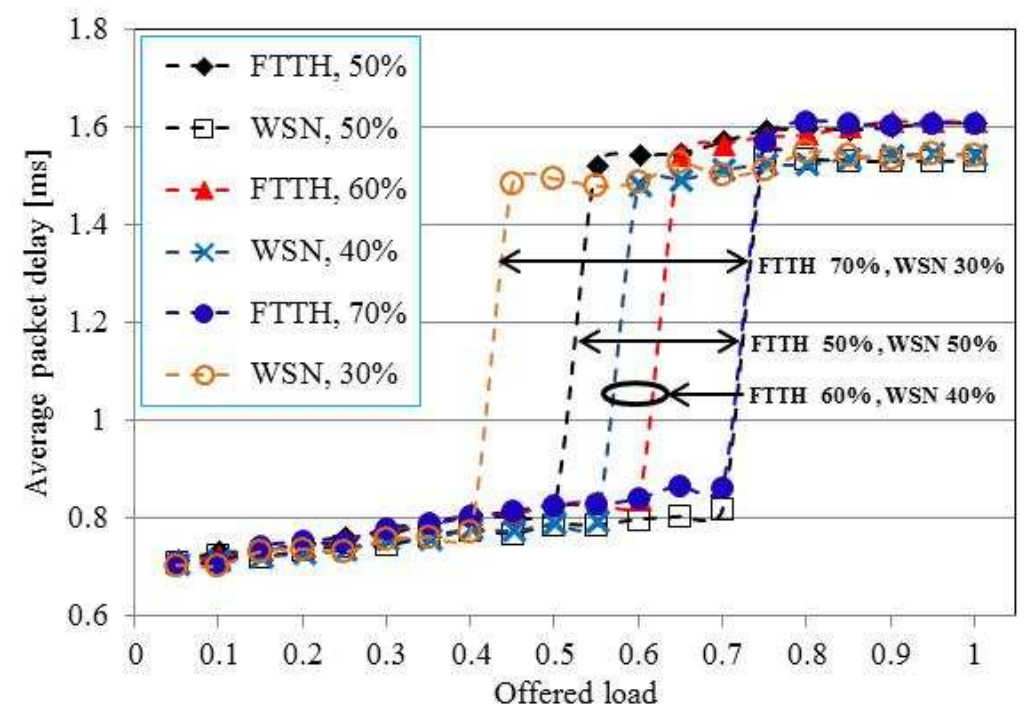

Figure 10. Average packet delay for uniform traffic load with the LS scheme

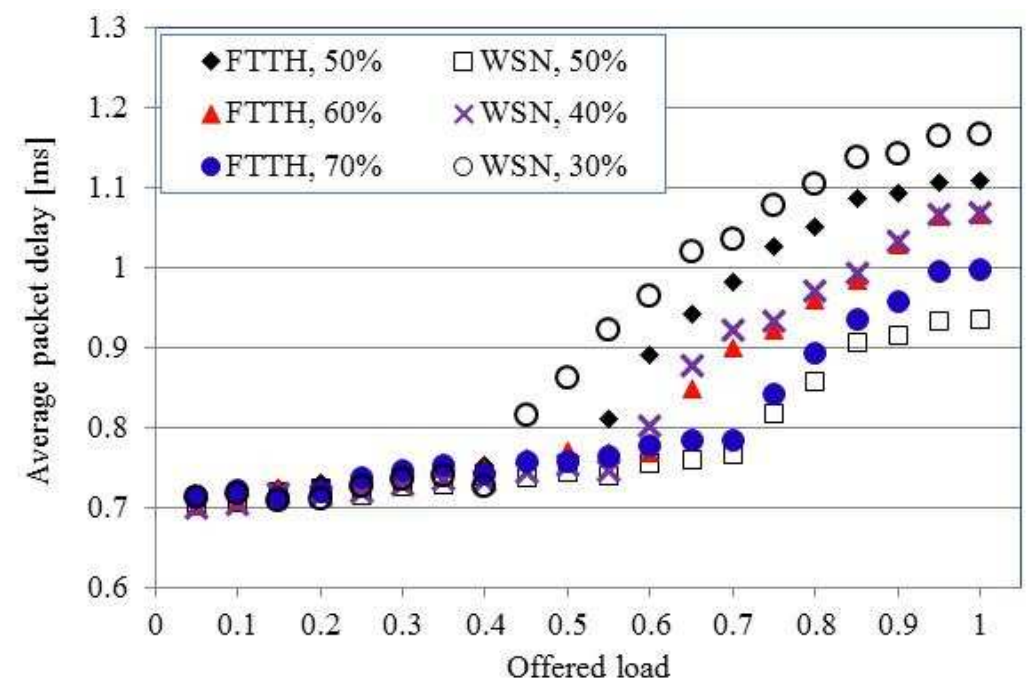

Figure 11. Average packet delay for non-uniform traffic load with the LS scheme 
Figure 11 shows the average end-to-end packet delay vs. offered load characteristics under nonuniform traffic conditions using different bandwidth allocation ratios. From the results, it is clear that the optimized average packet delay for non-uniform traffic is also achieved at $60 \%$ and $40 \%$ bandwidth for FTTH terminals and CHs of WSN respectively.

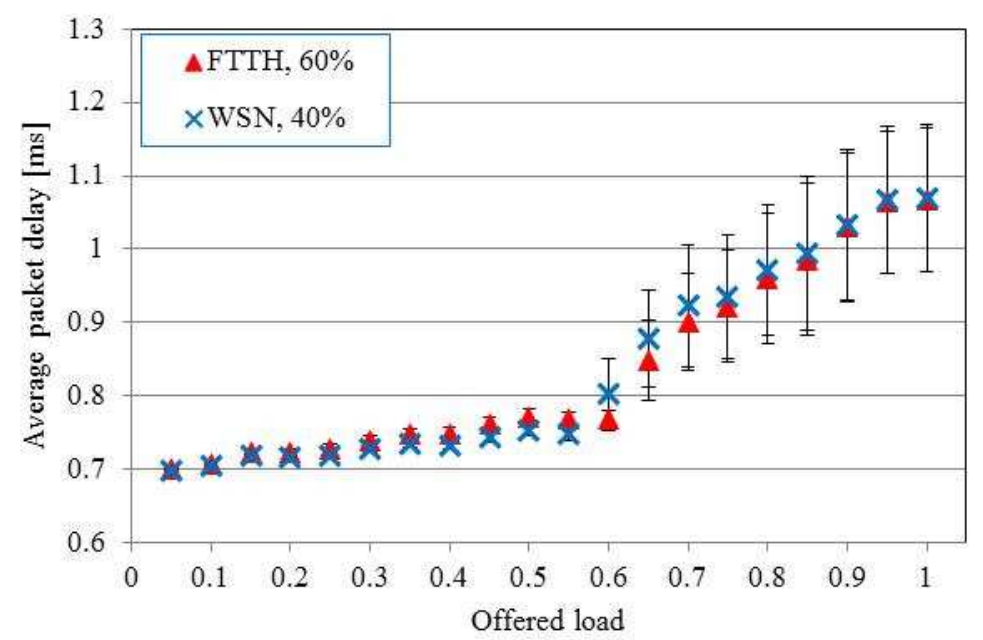

Figure 12. Analysis of error bars for non-uniform traffic load with the LS scheme

We provide the error bars analysis using standard deviation for non-uniform traffic condition for optimized (60\% bandwidth for FTTH terminals and $40 \%$ bandwidth for CHs of WSN) bandwidth allocation ratio among FTTH terminals and CHs of WSN of the multi-OLT PON. Figure 12 shows the average packet delay vs. offered load characteristic with error bars using standard deviation for non-uniform traffic condition with the LS bandwidth allocation scheme. From the analysis of error bars it can be mentioned that with the increase of offered load standard deviation is also increased. However, the maximum deviation is not more than $20 \%$ at maximum offered load of 1.0 .

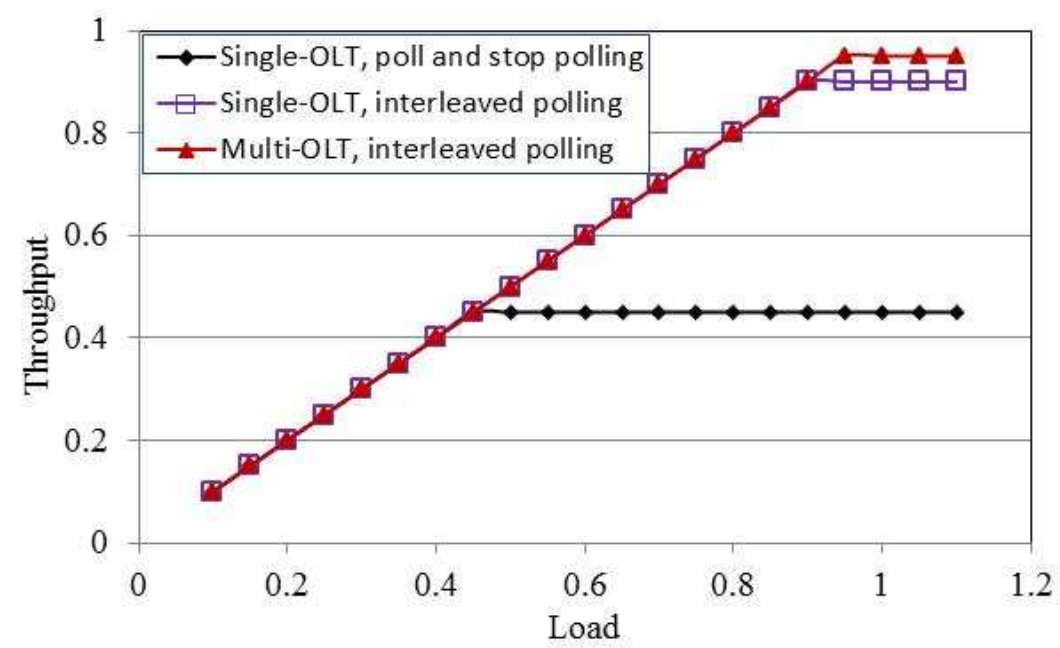

Figure 13. Comparison of throughput

Finally, we provide the comparison of throughput among the single-OLT polling algorithms and the multi-OLT interleaved polling algorithm. Figure 13 shows the throughput of the single-OLT poll and stop polling algorithm, the single-OLT interleaved polling algorithm, and the multiOLT interleaved polling algorithm. As shown in the figure, the single-OLT poll and stop polling algorithm has the lowest throughput (less than 50\%) due to the wastage of bandwidth as idle 
International Journal of Wireless \& Mobile Networks (IJWMN) Vol. 3, No. 6, December 2011

time, whereas the single-OLT and the multi-OLT interleaved polling algorithms overcome this bandwidth wastage problem to improve the channel utilization. Moreover, the multi-OLT interleaved polling algorithm achieves a throughput of 95\% (compared with the single-OLT interleaved polling algorithm having 90\%), which is due to the bandwidth savings by avoiding guard time between every two successive ONUs.

\section{Conclusions}

In this paper, we proposed a multi-OLT PON structure for both FTTH terminals and WSN. A modified interleaved polling algorithm and scheduling algorithm of a control message were also proposed. From the computer simulation results, evaluating average packet delay, it is found that the proposed structure can reduces the end-to-end latency about $0.1 \mathrm{~ms}$ up to the offered load of 0.6 while $0.2 \mathrm{~ms}$ at offered load of 1.0 for both uniform and non-uniform traffic loads. Moreover, the multi-OLT PON can accommodate 5\% more traffic load than the single-OLT PON without suffering from congestion. Therefore, it can be mentioned that the multi-OLT PON can effectively connects CHs of large WSN to make a converged network with FTTH terminals of a u-City with latency efficiency. The analysis performed in this study using the existing FS and LS bandwidth allocation schemes for both uniform and non-uniform traffic conditions proves the validity of the proposed multi-OLT PON structure. Moreover, the proposed multi-OLT PON structure with modified interleaved polling algorithm also outperforms other existing single-OLT polling algorithms in term of throughput for nonuniform traffic load with LS scheme. Furthermore, it is found that the convergence of FTTH and WSN in a multi-OLT PON is an efficient approach in that it provides cost effective solution than using two separate PONs, less packet delay, improved bandwidth utilization, and throughput under both uniform and non-uniform traffic conditions.

\section{ACKNOWLEDGEMENTS}

This work was supported in part by the JSPS-NRF bilateral joint research project.

\section{REFERENCES}

[1] Shinya Ito and Kenji Yoshigoe, (2009) "Performance Evaluation of Consumed-Energy-TypeAware Routing (CETAR) for Wireless Sensor Networks" International Journal of Wireless \& Mobile Networks (IJWMN), vol. 1, no. 2, pp 90-101.

[2] Shio Kumar Singh, M P Singh, and D K Singh, (2010) "Energy Efficient Homogenous Cluster ing Algorithm for Wireless Sensor Networks" International Journal of Wireless \& Mobile Networks(IJWMN), vol.2, no.3, pp 49-61.

[3] Jun Tang, Xinyu Jin, Yu Zhang, Xianmin Zhang, Wenyu Cai, (2007) "A Hybrid Radio Over Fiber Wireless Sensor Network Architecture," Proc. of International Conference on Wireless Communications, Networking and Mobile Computing,WiCom2007, pp 2675-2678.

[4] Monir Hossen, Byung-Jun Jang, Ki-Doo Kim, and Youngil Park, (2009) "Extension of Wireless Sensor Network by Employing RoF based 4G Networks," Proc. of the $11^{\text {th }}$ International Conference on Advanced Communication Technology(ICACT), pp 275-278 .

[5] Monir Hossen, Ki-Doo Kim, and Youngil Park, (2010) "Efficient Clustering Algorithm for Delay Sensitive PON-based Wireless Sensor Network," Proc. of International Conference on Optical Internet, pp 341-343.

[6] G. Kramer, B. Mukherjee, and G. Pessavento, (2002) "IPACT: A Dynamic Protocol for an Ethernet PON (EPON)," IEEE Communication Magazine, vol.40, issue 2, pp 74-80.

[7] Monir Hossen, Ki-Doo Kim, and Youngil Park, (2010) "Synchronized Latency Secured MAC Protocol for PON Based Large Sensor Network," Proc. of the $11^{\text {th }}$ International Conference on Advanced Communication Technology(ICACT), pp 1528-1532. 
International Journal of Wireless \& Mobile Networks (IJWMN) Vol. 3, No. 6, December 2011

[8] Masaki Tanaka, Takashi Nishitani, Hiroaki Mukai, Seiji Kozaki, and Hideaki Yamanaka, (2011) "Adaptive Dynamic Bandwidth Allocation Scheme for Multiple-Service in 10G-EPON System," Proc. of IEEE ICC 2011.

[9] G. Kramer and B. Mukherjee, (2001) "Ethernet PON: Design and Analysis of an Optical Access Network," Photonic Network Communication, Vol. 3, no. 3, pp 307-319.

[10] G. Kramer, B. Mukherjee, S. Dixit, Y. Ye, R. Hirth, (2002) "Supporting Differentiated Classes of Service in Ethernet Passive Optical Networks," Journal of Optical Networks, vol.1, no.8, pp 280-298.

[11] M. Ma, Y. Zhu, and T. H. Cheng, (2003) "A Bandwidth Guaranteed Polling MAC Protocol for Ethernet Passive Optical Networks," Proc. of IEEE INFOCOM, San Francisco, CA, pp 22-31.

[12] Chadi M. Assi, Yinghua Ye, Sudhir Dixit, Mohamed A. Ali, (2003) "Dynamic Bandwidth Allocation for Quality-of-Service Over Ethernet PONs," IEEE Journal on Selected Areas in Communications, vol. 21, no.9, pp 1467-1477.

[13] IEEE Draft P802.3ah ${ }^{\mathrm{TM}} / \mathrm{D} 1.2$, (2002) "Media Access Control Parameters, Physical Layers and Management Parameters for Subscriber Access Networks," http://grouper.ieee.org/groups/802/3/efm.

[14] Bjorn Skubic, Jiajia Chen, Jawwad Ahmed, Lena Wosinska, and Biswanath Mukherjee, (2009) "A Comparison of Dynamic Bandwidth Allocation for EPON, GPON, and Next-Generation TDM PON" IEEE Communication Magazine, pp S40-S48.

[15] I-Shyan Hwang, Zen-Der Shyu, Liang-Yu Ke, Chun-Che Chang, (2008) " A Novel Early DBA Mechanism with Prediction-based Fair Excessive Bandwidth Allocation Scheme in EPON," Journal of Computer Communications, vol. 31, no. 9, pp 1814-1823.

[16] B. Lannoo, L. Verslegers, D. Colle, M. Pickavet, P. Demeester, and M. Gagnaire, (2007) "Thorough Analysis of the IPACT Dynamic Bandwidth Allocation Algorithm for EPONs," Proc. of Fourth International Conference on Broadband Communications, Networks and Systems, pp 486-494.

[17] Kyuho Son, Hyungkeun Ryu, Song Chong, (2004) "Dynamic Bandwidth Allocation Schemes to Improve Utilization Under Non-uniform Traffic in Ethernet Passive Optical Networks," Proc. of IEEE International Conference on Communications, vol. 3, pp 1766-1770.

[18] V. Rajendran, K. Obraczka, and J.J. Garcia-Luna-Aceves, (2006) “ Energy-Efficient, CollisionFree Medium Access Control for Wireless Sensor Networks," Journal of Wireless Networks, vol. 12, no. 1 , pp 63-78.

Monir Hossen received his B. Sc. in Electrical and Electronic Engineering from Khulna University of Engineering and Technology (KUET), Bangladesh in 2002. Then, he joined as a faculty member in the Electronics and Communication Engineering department of KUET in 2004. He completed his M. Sc. in Electronics Engineering from Kookmin University, Korea in 2010. Currently, he is working toward the $\mathrm{PhD}$ degree in Interdisciplinary Graduate School of Medicine and Engineering, University of Yamanashi, Japan. His present research interest focus on PON based FTTH and wireless sensor networks.

Dr. Masanori Hanawa received the B.E., M.E., and PhD degrees all from Saitama University, Japan, in 1990, 1992, and 1995, respectively. In 1995, he joined in the Faculty of Engineering, Yamanashi University, Japan as a research associate. Since 2002, he has been an associate professor of the university. Current his affiliation is the Interdisciplinary Graduate School of Medicine and Engineering, the national university corporation, University of Yamanashi, Japan. His research interests are in ultra-wideband impulse radio systems including radars for medical applications or landmine detection, optical fiber communications, optical signal processing based on optical transversal filtering, and optical code division multiplexing.
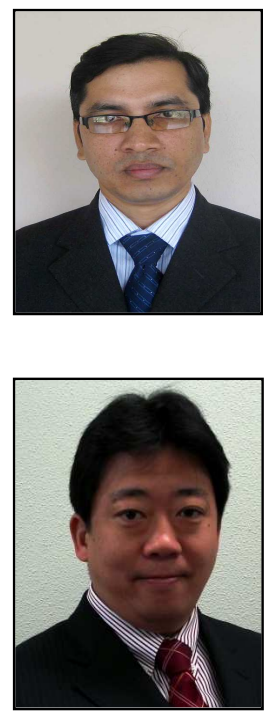